\title{
Evolution, geographic spreading, and demographic distribution of Enterovirus D68
}

\author{
Emma B. Hodcroft, ${ }^{1,2, *}$ Robert Dyrdak, ${ }^{3,4}$ Cristina Andrés, ${ }^{5}$ Adrian Egli, 6,7 Josiane Reist, ${ }^{6,7}$ Diego García \\ Martínez de Artola, ${ }^{8}$ Julia Alcoba Flórez, ${ }^{8}$ Hubert G. M. Niesters, ${ }^{9}$ Andrés Antón, ${ }^{5}$ Randy Poelman, ${ }^{9}$ Marijke \\ Reynders, ${ }^{10}$ Elke Wollants, ${ }^{11}$ Richard A. Neher, ${ }^{1,2}$ and Jan Albert ${ }^{3,4}$ \\ ${ }^{1}$ Biozentrum, University of Basel, Basel, Switzerland \\ ${ }^{2}$ Swiss Institute of Bioinformatics, Basel, Switzerland \\ ${ }^{3}$ Department of Clinical Microbiology, Karolinska University Hospital, Stockholm, Sweden \\ ${ }^{4}$ Department of Microbiology, Tumor and Cell Biology, Karolinska Institute, Stockholm, Sweden \\ ${ }^{5}$ Respiratory Viruses Unit, Virology Section, Microbiology Department, Hospital Universitari Vall d'Hebron, \\ Universitat Autónoma de Barcelona, Vall Hebron Research Institute, Barcelona, Spain \\ ${ }^{6}$ Clinical Bacteriology and Mycology, University Hospital Basel, Basel, Switzerland \\ ${ }^{7}$ Applied Microbiology Research, Department of Biomedicine, University of Basel, Basel, Switzerland \\ ${ }^{8}$ Department of Clinical Microbiology, Hospital Universitario Nuestra Señora de Candelaria, Tenerife, Spain \\ ${ }^{9}$ University of Groningen, University Medical Center Groningen, Department of Medical Microbiology, \\ Division of Clinical Virology, Groningen, The Netherlands \\ ${ }^{10}$ Unit of Molecular Microbiology, Medical Microbiology, Department of Laboratory Medicine, AZ Sint-Jan Brugge AV, \\ Bruges, Belgium \\ ${ }^{11}$ KU Leuven, Rega Institute, Department of Microbiology, Immunology and Transplantation, \\ Laboratory of Clinical \& Epidemiological Virology, Leuven, Belgium
}

\begin{abstract}
Background Worldwide outbreaks of enterovirus D68 (EV-D68) in 2014 and 2016 have caused serious respiratory and neurological disease.

Methods We collected samples from several European countries during the 2018 outbreak and determined 53 near full-length genome ('whole genome') sequences. These sequences were combined with 718 whole genome and 1,987 VP1-gene publicly available sequences.

Findings In 2018, circulating strains clustered into multiple subgroups in the B3 and A2 subclades, with different phylogenetic origins. Clusters in subclade B3 emerged from strains circulating primarily in the US and Europe in 2016, though some had deeper roots linking to Asian strains, while clusters in A2 traced back to strains detected in East Asia in 2015-2016. In 2018, all sequences from the USA formed a distinct subgroup, containing only three non-US samples. Alongside the varied origins of seasonal strains, we found that diversification of these variants begins up to 18 months prior to the first diagnostic detection during a EV-D68 season. EV-D68 displays strong signs of continuous antigenic evolution and all 2018 A2 strains had novel patterns in the putative neutralizing epitopes in the BC- and DE-loops. The pattern in the BC-loop of the USA B3 subgroup had not been detected on that continent before. Patients with EV-D68 in subclade A2 were significantly older than patients with a B3 subclade virus. In contrast to other subclades, the age distribution of A2 is distinctly bimodal and was found primarily among children and in the elderly.

Interpretation We hypothesize that EV-D68's rapid evolution of surface proteins, extensive diversity, and high rate of geographic mixing could be explained by substantial reinfection of adults.

Funding University of Basel and Swedish Foundation for Research and Development in Medical Microbiology
\end{abstract}

\section{INTRODUCTION}

Enterovirus D68 (EV-D68) has caused worldwide outbreaks of serious respiratory and neurological disease in 2014 and thereafter. In particular, EV-D68 infection has been associated with acute flaccid myelitis (AFM) (Hixon et al. , 2019). EV-D68 was first isolated in 1962, (Schieble et al., 1967 ) but rarely reported until the recent outbreaks (Centers for Disease Control and Pre-

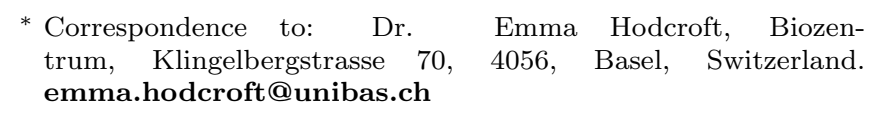

vention (CDC), 2011, Holm-Hansen et al., 2016, Khetsuriani et al., 2006; Rahamat-Langendoen et al., 2011). Yet, an almost ubiquitous presence of specific neutralizing antibodies indicates that infection with the virus has been very common before the recent outbreaks (Vogt and Crowe Jr, 2018).

In many countries, particularly in Europe and North America, EV-D68 has exhibited a biennial pattern, with peaks in the late summers and autumns of evennumbered years $(2014,2016,2018)$ (Khetsuriani et al. 2006; Kramer et al., 2018, Messacar et al., 2019; Poelman et al., 2015b; Pons-Salort et al., 2018; Shen et al. 2019: Uprety et al. 2019). Other countries have re- 
ported odd-year outbreaks, such as Thailand in 20092011 (Linsuwanon et al. 2012), Australia in 2011 and 2013 (peaking in Southern Hemisphere winter to spring) (Levy et al., 2015), and Japan in 2015 (Funakoshi et al. 2019). In 2018, circulation was reported from Europe in Wales (Cottrell et al., 2018), Italy (Pellegrinelli et al. 2019), France (Bal et al. 2019), and in the US (Kujawski et al. 2019, Messacar et al., 2019).

Phylogenetic analysis of EV-D68 sequences has revealed extensive diversity (Tokarz et al. 2012). This diversity is grouped into the major clades $\mathrm{A}, \mathrm{B}$, and $\mathrm{C}$, with a most recent common ancestor (MRCA) in the mid-1990s. Clades A and B are further divided into subclades A1, A2, B1, B2, and B3. Most previous phylogenetic analyses have focused on the capsid protein VP1 sequence (Du et al., 2015; Tokarz et al., 2012). VP1 is one of the more variable proteins and contains important receptor binding sites and putative neutralizing epitopes, such as the hypervariable BC- and DE-loops. Different subclades differ at several positions in these loops, and previous studies show that they have an elevated rate of amino acid substitutions (Dyrdak et al. 2019) and appear to be under positive selection (Du et al. | 2015; Dyrdak et al., 2019, Imamura et al., 2014, Lau et al., 2016, Linsuwanon et al. (2012).

The great majority of reported EV-D68 cases are pediatric (Holm-Hansen et al., 2016), and sero-positivity to EV-D68 increases rapidly during childhood, reaching ubiquity in adults (Harrison et al. 2019 Karelehto et al. 2019, Sun et al. 2018, Xiang et al. 2017). Relatively little is known about immunity to EV-D68, but infection appears to elicit long-lasting strain-specific immunity, as evidenced by the high prevalence among adults of neutralising antibodies to the prototype Fermon strain from 1962 (Harrison et al. 2019; Karelehto et al. 2019: Smura et al. 2010: Xiang et al. | 2017). While some studies indicate strain-specific differences in neutralizing titers in different age-groups, the degree to which EV-D68 evolution is driven by immune escape or whether incidence patterns are determined by pre-existing immunity is unclear.

Here, we report sequences from samples collected across Europe in late-summer and autumn 2018 and combine them with publicly available data to comprehensively investigate the global phylodynamics and phylogeography of EV-D68. These data and phylogenies are available on the Nextstrain platform at nextstrain.org/enterovirus, which provides a comprehensive phylogenetic analysis pipeline, a visualization tool and a public web-interface (Hadfield et al. 2018).

\section{MATERIALS \& METHODS}

The study aimed to characterize the genetic diversity and geographic distribution of EV-D68 variants in Eu- rope during the 2018 season and to compare these variants with EV-D68 variants collected earlier across the world. Respiratory samples $(\mathrm{n}=55)$ that had tested positive for EV-D68 were obtained from six clinical virology laboratories (Stockholm, Sweden; Groningen, the Netherlands; Leuven, Belgium; Barcelona, Spain; Basel, Switzerland; and Tenerife, Canary Islands, Spain). For a more detailed description of screening, isolation, and ethical approvals, see supplementary methods I.A.

Samples were collected from 29 Aug to 28 Nov 2018 (mean 4 Oct, median 1 Oct, interquartile range $22 \mathrm{Sept}$ to 15 Oct, see Supp. Fig. 11). Thirty-two of the 53 patients were male $(60.4 \%)$. The median age was 3.0 years (mean 20.8 years, range 1 month to 94 years), with a majority of children being five years or younger (34 of 36 children), and a majority of adults being older than 50 years (13 of 17 adults). 39 of 43 patients with clinical information presented with repiratory illness as the main symptom. Of 38 patients with admission status information, 32 were hospitalized ( 5 admitted to an intensive care unit (ICU)) and 6 were out-patient. There were no records of AFM among the study patients. Detailed information about the samples is available in Supp. Table I.

Near full-length genomes (NFLG) were obtained by sequencing four overlapping fragments on the Illumina next-generation sequencing (NGS) platform as described previously (Dyrdak et al. 2019) (53 of 55 samples were successfully sequenced and included in the further analyses). Sequences were aligned with and annotated according to the 1962 Fermon strain (GenBank accession AY426531). See supplementary methods I.B for details.

We combined the new 53 EV-D68 genomes with sequences available from GenBank and consolidated available metadata. This included 718 genome sequences with length $\geq 6,000 \mathrm{bp}$ and 1,987 sequences covering $\geq 700 \mathrm{bp}$ of the VP1 region of the genome (see I.C. We analyzed the combined sequence data set with the Nextstrain pipeline (Hadfield et al., 2018). The detailed analysis workflow is described in supplementary methods I.E.

\section{Role of the funding source}

The funding sources had no involvement in any part of this study. The corresponding author had full access to all the data in the study and had the final responsibility for the decision to submit for publication.

\section{RESULTS}

\section{Interactive near real-time phylogenetic analysis with Nextstrain}

To enable global genomic surveillance of EV-D68, we implemented an automated phylogenetic analysis pipeline using Nextstrain which generates an interactive 

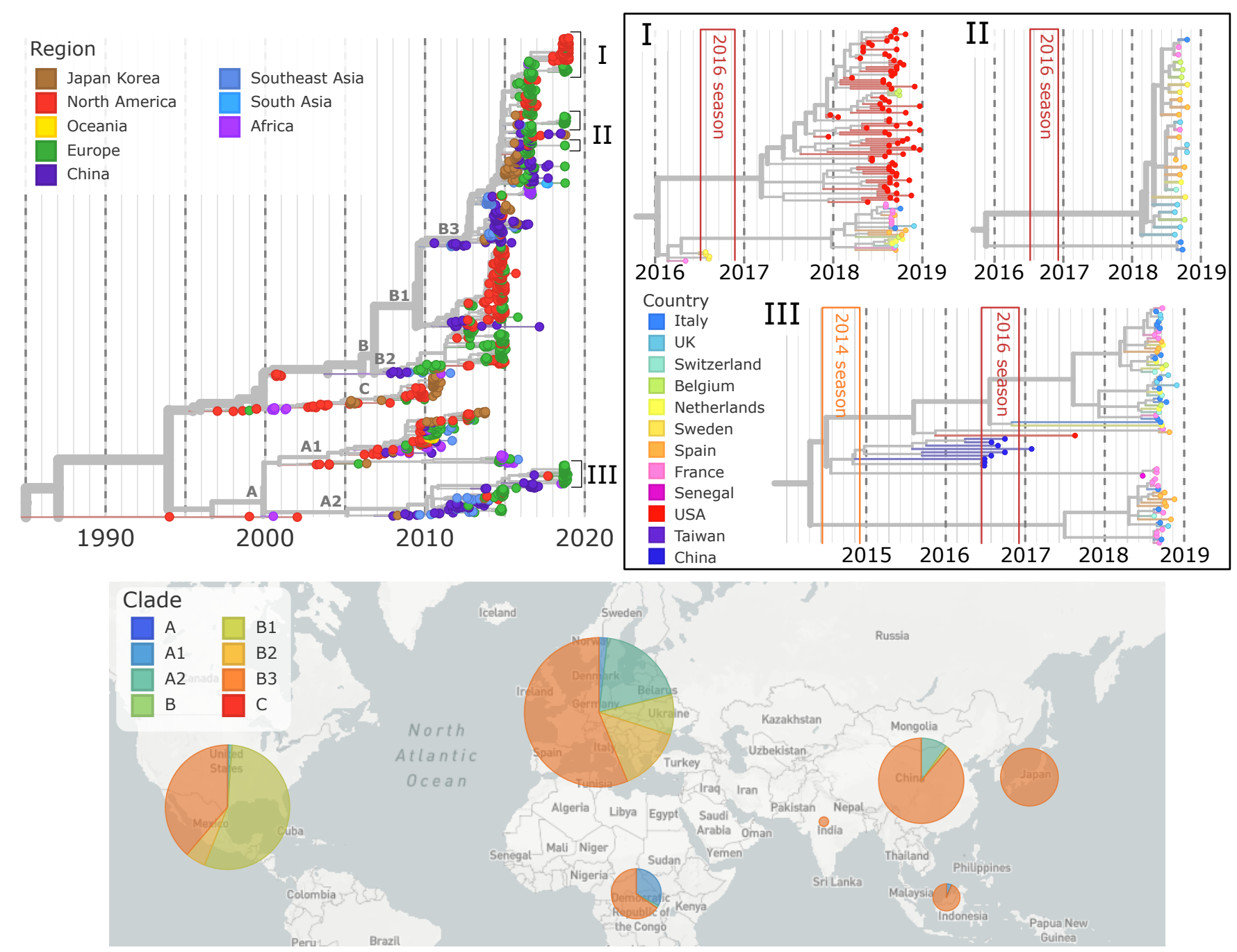

FIG. 1 Time-scaled phylogeny of Enterovirus D68. A time-scaled phylogeny of VP1 segments, colored by region, is shown top-left. Key clusters (I, II, III) from the 2018 season have been highlighted on the top-right, colored by country. To display these clusters at a high resolution, the whole genome phylogenies are used. The 2014 and 2016 EV-D68 seasons are shown in orange and red boxes. Below, a map shows the distribution of subclades by region from 2014-2018.

visualization integrating a phylogeny with sample metadata such as geographic location or host age. This analysis is available at nextstrain.org/enterovirus and was updated whenever new data became available and we intend to keep it up-to-date going forward. This rolling analysis revealed a dynamic picture of diverse clades of EV-D68 circulating globally.

Fig. 11 shows a time-scaled phylogenetic tree of all available VP1 sequences ( $\geq 700 \mathrm{bp}$ ) collected since 1990. Since 2014 , the global circulation of EV-D68 has been dominated by viruses from the B1 and B3 subclades, with A1 and A2 accounting for about 5 to $30 \%$ of viruses sampled in China, Europe, and Africa (see bottom of Fig. 1 and Fig. 3B). Both our 53 new sequences and other available EV-D68 variants from 2018 grouped into the B3 and A2 subclades.

\section{Multiple independent origins of the 2018 season}

As shown in Fig.11, VP1 sequences collected in 2018 fell into multiple distinct subgroups with MRCAs in 2016 or more recently. Six of these subgroups and one singleton fell into subclade B3, while another four subgroups were part of subclade A2. The largest B3 subgroup (zoomed at the top of Fig. 1) consisted of 80 samples from the USA and 3 Belgian samples, while most remaining subgroups were smaller and dominated by European samples.

The B3 subgroups from 2018 mostly originate from within the EV-D68 sequence diversity that circulated during the 2016 outbreak. This is expected as the B3 subclade was well-sampled in Europe, North America, China, and Japan during 2015-2016. The 2018 subgroups did not necessarily have ancestors in 2016 from the same geographic regions. Instead, some subgroups sampled in 
Europe in 2018 had their closest relatives in 2016 in Asia, and vice versa. All 2018 A2 subclade samples traced back to strains circulating in China and Taiwan in 2015-2016. These observations suggest relatively rapid global mixing of EV-D68.

\section{Undetected EV-D68 diversification}

Our phylogenetic analysis suggests that many of the 2018 subgroups started to diversify as early as mid-2017 but were not sampled until about one year later. Both of the large, predominantly European 2018 subgroups have estimated MRCAs in the middle to end of 2017, with extensive diversification until the first samples were collected in August of 2018. The large American 2018 subgroup began diversifying in early 2017. Though the ancestors of these subgroups must have been circulating, there are no corresponding samples. This pattern is not unique to the B3 subclade or to 2018. Similar patterns of 'hidden' diversification can be seen in the two large 2018 clusters in the A2 subclade, and in the 2014 and 2016 outbreaks, with the ancestors of samples taken during these outbreaks beginning to diversify up to a year beforehand.

We quantified this diversification over time in Fig. $2 \mathrm{~B}$, where the number of lineages which lead to the samples taken during each season is plotted over time (Nee et al. 1995) (see supplementary methods I.F). Despite a variable number of lineages present at the end of each outbreak (363 for 2014, 119 for 2016, and 122 for 2018), each season traces back to only 10-18 lineages two years prior, reflecting a marked diversification which began about 18 months before the first sequenced samples of each season were collected. Four years prior to the outbreak year the number of lineages dropped further, to 2-7, representing deep splits in the tree.

When the change in number of lineages is plotted alongside sampling times (Fig. $2 \mathrm{C}$ ), peak diversification (black) appears before peak sampling periods (purple). Both plots illustrate that the majority of diversification occurs prior to when samples are taken (i.e. 'hidden' diversification), and slows down during the outbreak. This pattern is consistent with the expectation that lineages split in expanding populations and little coalescence is observed when populations are large (Kingman, 1982). In the 2014 and 2018 seasons, the majority of diversification immediately preceded the sampling period, evident in the steep slopes in the first half of the outbreak year in Fig. 2B, and in the sharp peaks during the outbreak year in Fig. 2 2 C). In the 2016 outbreak, however, the diversification appears to have been slower, shown by the shallower but more stable slope in Fig. $2 \mathrm{~B}$ and the broad peak of lineage change in Fig. 2 $\mathrm{C}$. These patterns are recapitulated in the inferred pattern of the per-lineage coalescence rate through time ('skyline,' see Supp. Fig. 3.
The well-sampled seasons in 2014, 2016, and 2018 show a common pattern: diversity within an outbreak traces back to 10-18 lineages 2 years prior and 2-7 lineages 4 years before the outbreak.

Overall, our results suggest that EV-D68 diversity is maintained through multiple outbreak years by significant unreported year-round circulation.

\section{Migration of EV-D68 between countries and continents}

The relationship between 2016 and 2018 viruses suggested that EV-D68 transmits and migrates rapidly enough that viruses sampled in one continent in 2018 often have ancestors detected in 2016 on different continents. To quantify the geographical mixing we investigated the viral migrations between countries and continents during the comparatively well-sampled outbreaks of 2014, 2016 and 2018 (see supplementary methods I.F).

Of the 10 outbreak clusters in 2018, all but two were predominantly European. Within these clusters, strains from different European countries were thoroughly intermixed, with little sign of within-country clustering (see insets in Fig. 1). Consistent with this qualitative observation, the maximum likelihood estimate of the overall migration rate between European countries in 2018 was approximately $2 /$ year. Repeating the same analysis on the 2014 and 2016 outbreaks yielded about 2- to 3fold lower estimates, possibly due to less representative sampling in these years. Discrete trait analyses make a number of assumptions and the migration rate estimates should be viewed as an empirical summary of transitions observed on the tree rather than unbiased quantitative estimates.

At the level of broader geographic regions, mixing was less rapid. This is qualitatively evident from the clustering of regions in the phylogeny in Fig. 1 . The maximum likelihood migration rate between Europe, North America, and China estimated using a discrete state model fitted to years 2014-2018 was found to be $0.24 /$ year. This means that any given lineage has a chance of about 1 in 4-5 to switch regions in one year. After the two year inter-outbreak interval, about half the viral lineages would be expected to have spread to other geographic regions. Notably, though samples from the 2014 and 2016 outbreaks showed considerable mixing between European and North American sequences (Dyrdak et al. 2019), all 77 samples from the US in 2018 formed one distinct subgroup, containing only three samples from elsewhere (Belgium). Since the subgroup dominating the US in 2018 harbors substantial diversity and since both the US and Europe have been thoroughly sampled, these divergent patterns are difficult to explain with sampling biases alone. 

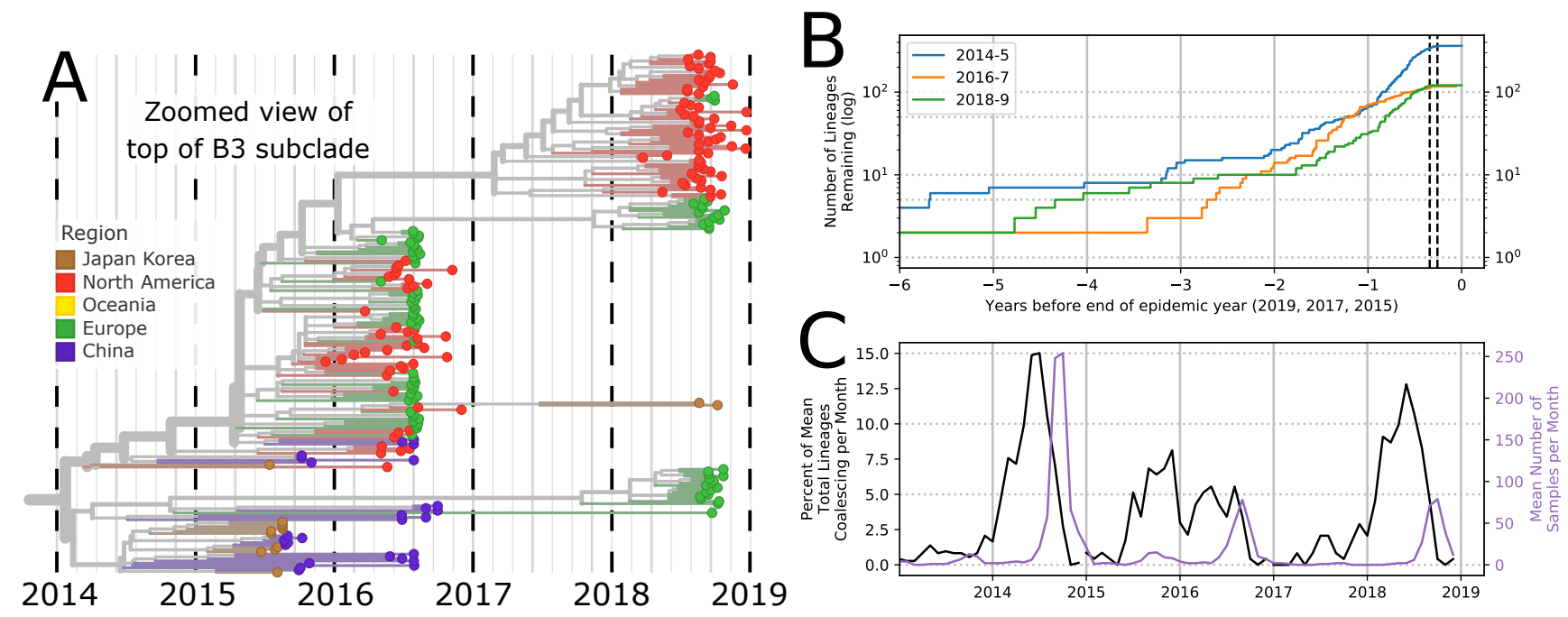

FIG. 2 Persistence and diversification of EV-D68 since 2014. Multiple EV-D68 lineages persist from one biennial outbreak to another. (A) Zoomed in view of the whole genome EV-D68 tree (for better time-resolution), showing 2018 subgroups in the B3 subclade. (B) Lineages which lead to the samples in the 2014, 2016, and 2018 seasons diversify over time. The dotted black lines show the IQR of all samples taken during 2014, 2016, and 2018. (C) The change in number of lineages (as $\%$ of total lineages) per month for each season (left y-axis) and the mean number of samples per month (right y-axis).
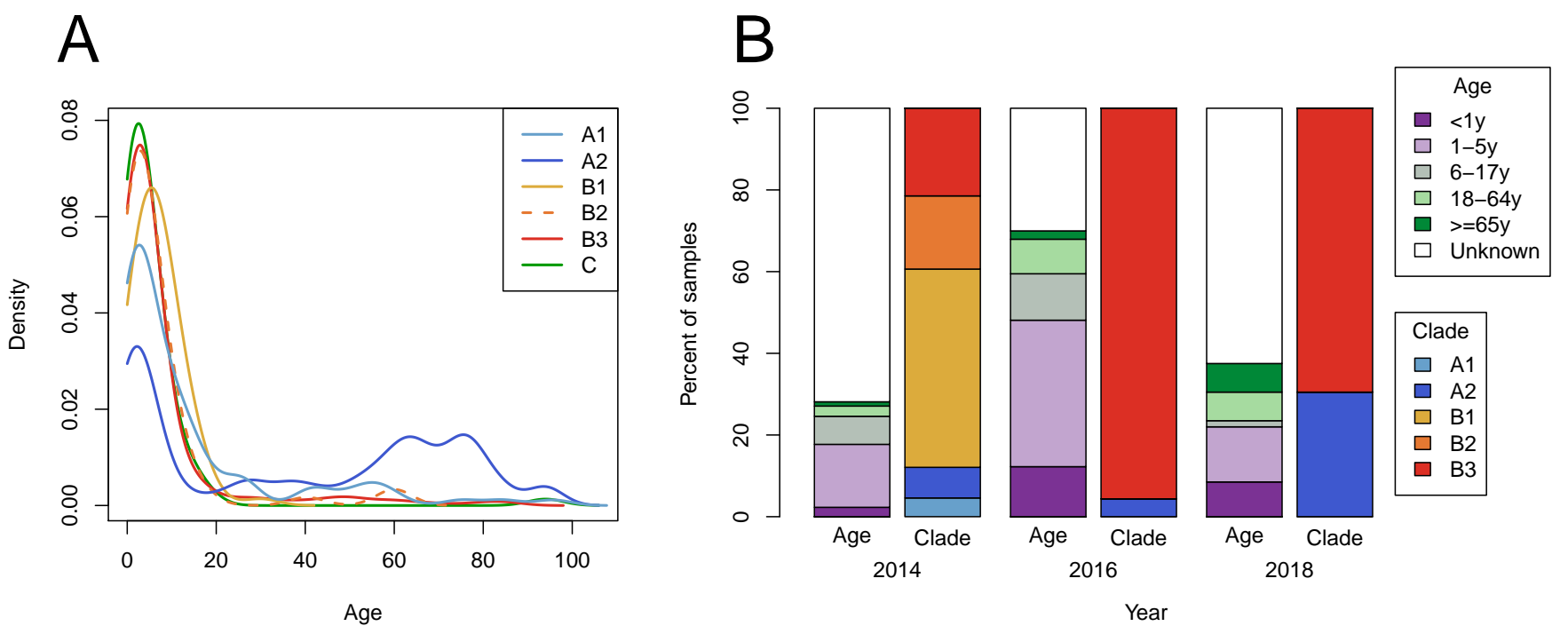

FIG. 3 Age distribution of EV-D68 samples. (A) The cumulative distribution over age separated by subclade; A2 being significantly more often detected in older persons. (B) The age of patients and subclade of their sample, for samples taken during 2014, 2016, and 2018 for which 'age range 1' was available.

\section{Subclade $A 2$ is over-represented in elderly}

We noted that many samples in A2 subclade were obtained from adults and the elderly, in contrast to other subclades. Similar observations have been made in some previous studies (Bal et al., 2019, Bottcher et al., 2016, Lau et al., 2016; Schuffenecker et al., 2016). Therefore, we investigated the age distribution in different subclades using a comprehensive data set of 743 samples with exact (non-range) age data and unambiguous subclade desig- nation (see supplementary methods I.D). Almost all sequences assigned to $\mathrm{B}$ or $\mathrm{C}$ clades were sampled from children, while subclade A2 viruses showed a bimodal age distribution (Fig. 3A), with $40 \%$ of A2 samples from children and $39 \%$ from the elderly (>60 years old). Subclade A1 was sampled predominantly in children with a minority of sequences sampled from individuals between 20 and 60 years of age. The distribution of age categories in each subclade is shown in Supp. Fig. 4

These associations are confirmed by statistical analy- 
sis. Both the A1 and A2 subclades were associated with significantly older ages compared to B3 subclade (linear model, $\mathrm{p}=0.001$ and $\mathrm{p}<0.001$, respectively), whereas individuals infected with $\mathrm{B} 2, \mathrm{~B} 1$, and $\mathrm{C}$ subclades did not have significantly different ages (Table I). While these p-values might by inflated due to regional differences in sampling different age groups, the difference in age distribution between the A2 and B subclades was robust to removal of countries by bootstrap analysis (Supp. Fig. 22) In addition to the age differences between the $\mathrm{A}$ and $\mathrm{B}$ subclades, the age distributions of the A1 and A2 subclades also differed significantly $(p<0.001$, KolmogorovSmirnov test).

The higher prevalence of the A2 subclade in 2018 as compared to previous outbreaks results in a significant skew towards older age groups in 2018 compared to 2014 and 2016 (linear model, $\mathrm{p}=0.005$ and $\mathrm{p}=0.001$, respectively) (Fig. 3 B).

\section{Antigenic evolution of EV-D68}

Previous molecular analyses of EV-D68 showed the BC- and DE-loops in the VP1 gene have elevated rates of amino acid substitutions (Dyrdak et al. 2019$)$ and indications of positive selection (Du et al., |2015 | Imamura et al., 2014, Lau et al., 2016, Linsuwanon et al., 2012). We examined the sequences of these putative neutralizing antibody targets (Liu et al., 2015a) over time, comparing the sequences of the loops at the root of the tree in 1990, at the base of the A and B clades, and from several sequences sampled in 2018 (Fig. 4B). Both loops have changed their sequence multiple times in each of these comparisons.

Still, even outside of these loops the surface of the virus has changed rapidly, suggesting further antigenic evolution. Fig. $4 \mathrm{~A}$ shows the 5 -fold symmetric arrangement of the crystal structure of the virus capsid protomer consisting of VP1, VP2, VP3, and VP4 (Liu et al., 2015b). Much of the virion surface is variable (panel A, subunit 5), and almost all variable residues in VP1 and VP2 are in surface exposed patches of the genome (panel C). A particularly dense cluster of variable positions is formed by the C-terminus of VP1 and central region of VP2. The C-terminus of VP1 was identified by Mishra et al. (2019) as a potential EV-D68 specific epitope.

The epitope turnover dynamics are more readily apparent when integrated with the phylogenetic tree, and trees colored by the epitope patterns found in the BC- and DEloops reveal signs of rapid and putatively antigenic evolution in EV-D68 (Fig. 4D \& E). The BC-loop in the A2 subclade, for example, has changed 3 times since 2010. All 2018 A2 samples have novel epitope patterns in the BC-loop (the two small grey 'other' clusters also have distinct novel patterns). The predominantly American B3 2018 subgroup also has novel BC-loop patterns, mostly pattern 'DTTQTF'. A total of 144 of 197 samples from 2018 have BC-loop sequences different from those observed in 2016 or earlier. The DE loop has undergone less rapid change in the recent past, but all major clades differ in their DE-loop sequence. The predominant epitope pattern in the mostly-American B3 subgroup ('NGSNNNTTYV') was present in 25 European sequences in 2016, but does not appear to have previously been seen in the USA. Notably, the B1 and B3 variants dominating the large outbreaks in 2014 and 2016 show little variation at the BC- nor DE-loops. Supp. Fig. 5 plots the mean number of mutations in the BC- and DE-loops acquired over time and shows that different subclades have acquired mutations at different rates. The patterns of molecular evolution revealed here are compatible with the notion of rapid antigenic evolution with rapid turnover of epitopes and frequent parallel changes in different clades, as observed for seasonal influenza viruses (Petrova and Russell, 2018).

TABLE I Age and Clade/Subclade

\begin{tabular}{|c|c|c|c|}
\hline (Sub)Clade & Number of Samples & Mean Age & Differs from Intercept \\
\hline B3 (Intercept) & 371 & 7.33 & \\
B2 & 59 & 6.54 & $N S(p=0.74)$ \\
B1 & 73 & 6.64 & $N S(p=0.75)$ \\
A2 & 79 & 38.13 & $* * * p<2 \times 10^{-16}$ \\
A1 & 88 & 13.66 & $* * p=0.002$ \\
C & 75 & 4.9 & $N S(p=0.25)$ \\
\hline
\end{tabular}

Linear model of subclade on age, showing the ages of patients in subclades A2 and A1 are significantly different from those in subclade B3. Subclades B2, B1, and C do not differ significantly from B3. Number of samples refers to the number of samples within a subclade for which exact age data was available.

\section{DISCUSSION}

Through a European multi-center collaboration and use of publicly available sequences and metadata in Genbank, we have shown that the circulation of EV-D68 in 2018 was not a single outbreak but consisted of smaller clusters of diverse viruses from the A2 and B3 subclades. We demonstrated a robust association of A2 viruses with infections in the elderly and provide evidence for rapid antigenic evolution of the EV-D68 surface proteins.

Recent EV-D68 circulation. We compared genetic diversity of viruses circulating in 2018 with those from the major US/European outbreaks in 2014 and 2016. In each of these seasons, sampled diversity traced back to 10-18 lineages at the time of the previous season two years earlier. Four to five years prior to a season, the number of ancestral lineages is reduced to 2-7. This suggests that EV-D68 has undergone waves of diversification about 1 year prior to the recognised outbreaks and that coalescence occured on a 2-4 year time scale. Similar pat- 
A

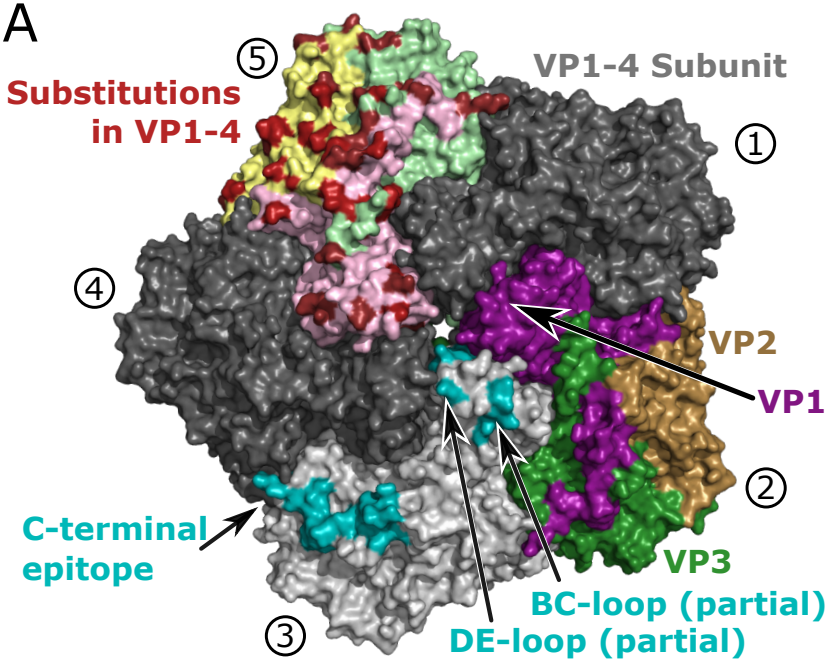

B

\begin{tabular}{|c|c|c|}
\hline & BC-Loop & DE-Loop \\
\hline Fermon & .A..S.G.... & $\ldots$ NND . . . \\
\hline NY93 & NHTSSDARTHKNFF & NGSSNSTYM \\
\hline A & $\ldots$........ & \\
\hline A2 & $\ldots$...E. VD..Y & $-\ldots N \ldots$ \\
\hline A1 & .A.E.Q.D... & $-\mathrm{S} \ldots \mathrm{T}$ \\
\hline B & D...A.Q.D... & $\ldots . . . N \ldots$ \\
\hline B3-US & D...T.Q.D... & $\ldots N . N \ldots V$ \\
\hline B3-EU & D....A.QAD .... & $\ldots$.....$V$ \\
\hline
\end{tabular}

Epitope At BC Loop (Pos 90,92,95,97,98,103)^

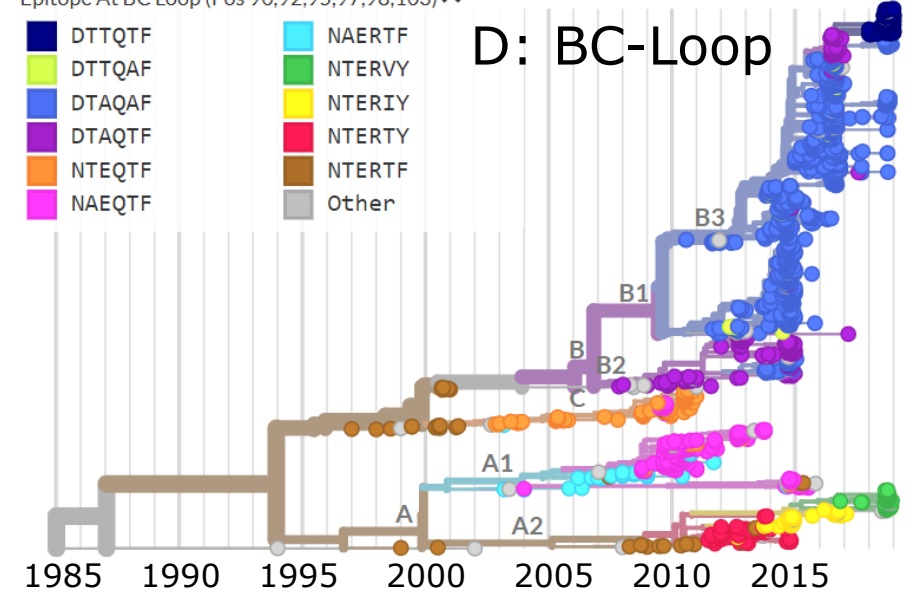

Epitope At DE Loop (Pos 140,141,142,143,144,145,146,149)

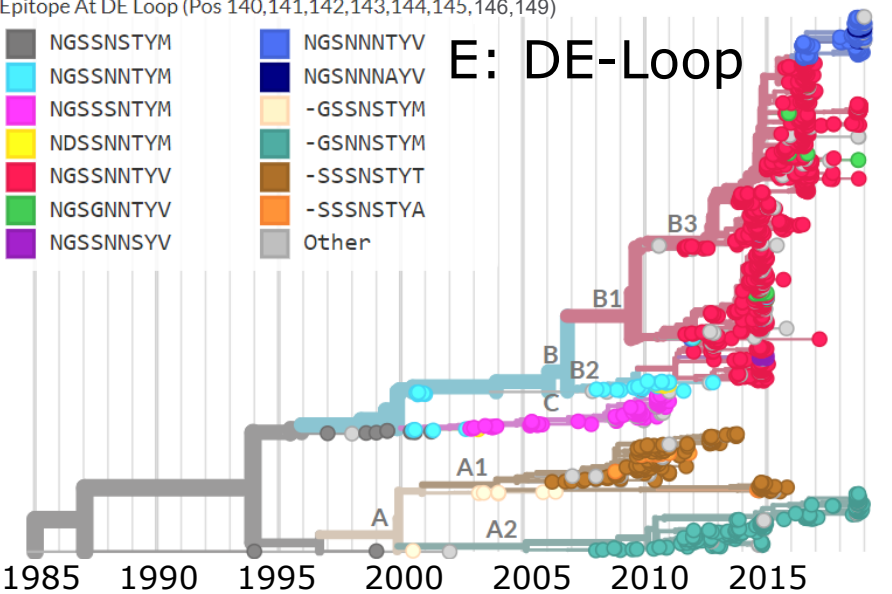

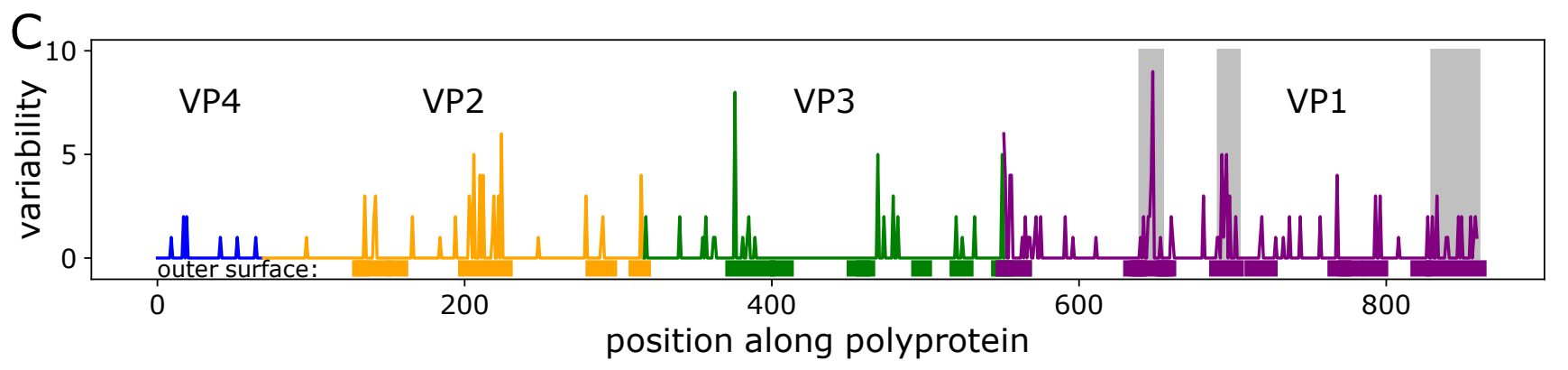

FIG. 4 Molecular evolution of the EV-D68 capsid. Panel A) Rendering of 5-fold symmetric arrangement of the crystal structure of the capsid protomer consisting of VP1, VP2, VP3 and VP4 (Liu et al., 2015b), using different copies of the protomer to highlight different aspects of the capsid organization, evolution, and immunogenicity. The five subunits are labelled 1-5 (circled numbers). Subunits 1 and 4 are present in dark grey to complete the structure. Subunit 2 shows the surface exposed proteins in purple (VP1), sand (VP2), and green (VP3), subunit 3 displays the different putative epitopes (Mishra et al. 2019) (note that the most variable parts of the BC- and DE-loops are not shown, as their structure is not resolved), and subunit 5 highlights variable positions in red, with the different proteins forming the surface indicated by pale colors. Panel B) The hypervariable BC- and DE-loops (partly missing from the structure in Panel A) in clusters and subclusters accumulated multiple changes since the root of the tree (the inferred root sequence patterns match those of the NY93 strain shown here). (BC-loop is AA positions 90-103; DE-loop is AA positions 140-148). Patterns for the C-terminus and a region of VP2 are shown in Supplementary Table II Panel C) Variable positions often coincide with surface exposed parts of the protein. Genes are highlighted by blue (VP4), orange (VP2), green (VP3), and purple (VP1). Grey boxes show, from left to right, the BC- and DE-loops, and C-terminus regions. Panels D\&E) Phylogenetic VP1 trees are colored by the most common epitope patterns of the 6 and 8 most variable amino-acid positions in the BC- and DE-loops, respectively. Particularly note-worthy is the rapid turnover of BC-loop variants in the recent evolution of the A2 subclade. Patterns for the C-terminus region are shown in Supp. Fig. 6 
terns can be observed for seasons prior to 2014, but the scarce sequence availability makes quantification of these patterns less reliable.

Geographic mixing. All major subgroups circulating in Europe in 2018 were sampled in multiple countries, despite the fact that the inferred $T_{M R C A}$ of most subgroups was only about one year prior to sampling. Consistent with this, we inferred rapid migration of lineages between European countries at rates of about 2/year. While the great majority of sequences in this dataset were obtained from respiratory samples, Majumdar et al. (2019) reported 27 EV-D68 sequences from wastewater samples from the UK (accession numbers MN018235MN018261). These sequences were well mixed with the European respiratory samples, indicating that the viruses detected in sampling in medical settings fairly accurately reflect the circulating strains.

At the level of continents or major geographic regions, migration was slower and accurate quantification difficult. Discrete trait analysis suggested migration rates between continents to be 5-10 fold lower than between European countries. While we observed rapid mixing between sequences sampled in the US and Sweden during the 2016 outbreak (Dyrdak et al., 2019), sequences sampled in the US in 2018 formed a largely US-specific cluster.

Despite the (currently) monophyletic origin of 2018 EV-D68 in the US, EV-D68 strains are remarkably wellmixed given that the majority of cases were in young children.

Age distributions. We undertook a thorough literature review and reached out to many authors to obtain information on the age of the sampled individuals. In total, we were able to assign ages to 743 sampled patients which allowed us to investigate age distributions with much higher accuracy than previous studies. We found that subclade A2 was sampled significantly more often in the elderly than other clades and subclades, which is consistent with results from previous smaller studies (Bal et al. 2019, Bottcher et al., 2016, Lau et al., 2016; Schuffenecker et al., 2016). More specifically, we found that A2 samples had a pronounced bimodal age distribution (Fig. 3A) with about $40 \%$ of the samples from children and $40 \%$ from the elderly above 60 years. Notably, the A1 subclade was also moderately over-represented in adults, but not specifically in the elderly.

The fact that almost all samples from the elderly population fell into the A2 subclade could have several reasons. (i) Individuals in this age group could be more prone to infection by A2 subclade viruses, possibly due to lower subclade-specific immunity. This possibility is supported by Harrison et al. (2019) who showed lower prevalence of neutralizing antibodies among adults to the A2 subclade than to B1, B2 and Fermon. (ii) Alternatively, A2 viruses are more virulent/symptomatic in elderly (irrespective of pre-existing immunity), but there are no data directly supporting this possibility.

Evidence for continuous antigenic evolution. Given that most EV-D68 cases are pediatric (HolmHansen et al. , 2016) and that almost everyone has high EV-D68 titers by the age of ten (Harrison et al. 2019 . Karelehto et al., 2019, Sun et al., 2018; Xiang et al., 2017), one might expect that selection for antigenic change is of minor importance for the molecular evolution of EV-D68. However, we found rapid evolution in putative neutralizing epitopes, including the previously studied BC- and DE-loops (Du et al. 2015; Dyrdak et al., 2019). In particular, the C-terminus of VP1 and a surface exposed patch in VP2 are accumulating amino acid substitutions at a high rate with substantial parallelism between clades. The C-terminus of VP1 was recently described as an EV-D68 specific immunoreactive epitope (Mishra et al. 2019). In the 3D-structure, the C-terminus of VP1 and VP2 forms a contiguous ridge, which is a plausible target for antibody binding that displays substantial differences between the A2 and B3 subclades.

Antigenic evolution is further supported by serology. Harrison et al. (2019) used a panel of sera collected in 2012 (prior to the large clade B outbreak in 2014) and measured neutralizing antibody titers against the prototypic Fermon strain from 1962 and against viruses from subclades B1, B2, and A2 sampled in 2014. Individuals older than 50 (i.e. born before 1962) have uniformly high titers against the Fermon strain (median $\log _{2}$ titer 10.5), while children below the age of 15 have low titers $\left(\log _{2}\right.$ titer 6 ). In contrast, titer distributions against representatives from subclades B1 and B2 were similar for individuals above the age of 5 (median between 7.8 and 9.5). Young children below the age 5, however, had markedly lower titers (median 5.8 (B1) and 3.2 (B2)) suggesting that a substantial fraction of those under 5 years old are still naive. In pregnant women tested from 1983 to 2002, all showed continuously high EV-D68 seroprevalence to Fermon, but on a population level titers declined over the study period (Smura et al. 2010). Taken together, this implies that individuals can retain high titers against a strain they were exposed to decades earlier (as shown by the titers of those over the age of 50 against Fermon, which has not circulated for many years), but that infection with recent EV-D68 strains does not elicit antibodies against Fermon - that is, the virus has evolved antigenically. Consistent with this, Imamura et al. (2014) showed that antisera raised in guinea pigs against viruses from subclades A2, B2, and C, did not neutralize Fermon.

Antigenic evolution could be driven by substantial re-infection of adults.

While EV-D68 has historically been primarily diagnosed in children, unrecognised (re-)infection of adults could explain two striking features of EV-D68 diversity and evolution: (i) consistent patterns of molecular evolution suggesting rapid immune-driven antigenic evolution 
(as observed in influenza A/H3N2 (Petrova and Russell, 2018)) and (ii) the rapid geographic mixing of different EV-D68 variants. The speed of antigenic evolution of influenza viruses has been negatively associated with the fraction of infection in children (Bedford et al. 2015), and adults travel much more than children, allowing more migration opportunities.

For pre-existing immunity to be a driving force of viral evolution, reinfection of older, previously exposed individuals, has to be sufficiently common. In adults with high antibody titers to EV-D68, post-outbreak titers against the circulating strain increase, implying both that existing immunity is not fully protective and that it can be boosted by subsequent re-exposure (Xiang et al. 2017). Adults are less likely to attend medical care than children and infections in adults may be milder, further reducing the likelihood that they are sampled and explaining why diagnoses and sequences come predominantly from children. Less-symptomatic infections in adults may be due to both physiology (e.g. children have smaller respiratory tracts) and partially protective immunity from previous EV-D68 infection.

Increases in EV-D68 infection in particular groups of adults, as we see in the A2 subclade and individuals over 60 , may be due to 'original antigenic sin,' whereby immune response is primarily based on the first pathogen encountered. If new EV-D68 strains evolve to be sufficiently distant from the initial EV-D68 strain that infected a particular age cohort, these individuals could be particularly vulnerable to re-infection and/or more severe infections.

While existing serological data are consistent with continuous antigenic evolution and frequent infection of adults, currently available serological studies typically include only a small number of EV-D68 strains (antigens), making systematic comparisons of preexisting immunity to different EV-D68 clades difficult.

Conclusion. Platforms like nextstrain.org allow the near real-time analysis and dissemination of sequence data integrated with relevant metadata, which we hope will be a valuable resource to the community. This has enabled our comprehensive analysis of new and existing EV-D68 data. We reveal a very dynamic picture of EVD68 circulation, characterized by rapid mixing at the level of countries within Europe, rapid evolution of surface proteins, and divergent and at times bimodal age distributions of different subclades. Combined with published serological data, our findings suggest a substantial contribution of adults in the global dispersion and continuous antigenic evolution of EV-D68.

\section{ACKNOWLEDGEMENTS}

We gratefully acknowledge expert sequencing service and advice by Tanja Normark, Cecilia Svensson, Anna Lyander, Emilia Ottosson Laakso and Valtteri Wirta at the Clinical Genomics Unit at Science for Life Laboratory (SciLifeLab), and expert technical assistance by Lina Thebo at the Karolinska Institute. The team at the University Hospital Basel thanks for excellent technical assistance of Daniela Lang. We would like to thank Moira Zuber for her work on implementing subclade specific mapping and Adam Mazur for his assistance in using PyMOL to generate the crystal structure in Figure 4

We are very grateful to researchers who shared their non-published age data with us: Amary Falls, Institut Pasteur de Dakar, Senegal; Sindy Böttcher \& Sabine Diedrich, Robert Koch Institute, Berlin, Germany; Katzumi Mizuta, Yamagata Prefectural Institute of Public Health, Yamagata, Japan; Sofie Midgley, Statens Serum Institut, Copenhagen, Denmark.

Analysis were run using sciCORE (scicore.unibas.ch) scientific computing core facility at University of Basel.

Finally, we are grateful to scientists and public health authorities globally for sharing EV-D68 sequences and metadata openly and without delay.

This study was funded by the University of Basel through core funding and a grant from the Swedish Foundation for Research and Development in Medical Microbiology.

\section{AUTHORS' CONTRIBUTIONS}

EBH, RAN, RD, and JA contributed to the study conception and design, interpreted the data, reviewed the relevant literature, and prepared the manuscript. $\mathrm{RD}$ and JA coordinated sample collection, selection, transport, and sequencing. EBH collected and curated publicly available data and scraped manuscripts for age data. $\mathrm{RD}$ contacted authors of previous publications to obtain further age information, curated study metadata, and conceptualized age and sampling figures. EBH and RAN assembled the sequences and performed the phylogenetic, migration, age, and antigenic evolution analyses. CA, AE, JR, DGMA, JAF, HGMN, AA, RP, MR, and EW coordinated ethics approval, metadata and sample collection, and sample testing, extraction, and shipping from their respective centers. All authors reviewed, edited, and commented on the draft manuscript and contributed to the final version.

\section{DECLARATION OF INTERESTS}

We declare no competing interests. 
TABLE II 2018 Samples Sequenced

\begin{tabular}{|c|c|c|}
\hline Lab & Country & Number of Samples \\
\hline Hubert Niesters et al. & Netherlands & 10 \\
Elke Wollants et al. & Belgium & 10 \\
Adrian Egli et al. & Switzerland & 6 \\
Andres Pagarolas et al. & Spain & 14 \\
Diego García Martínez de Artola & Spain & 8 \\
Jan Albert et al. & Sweden & 5 \\
\hline
\end{tabular}

This refers to samples included in analyses and may not include samples received that did not achieve sufficient coverage to be included.

\section{REFERENCES}

Altschul, S. F., W. Gish, W. Miller, E. W. Myers, and D. J. Lipman (1990), Journal of Molecular Biology 215 (3), 403

Andres, C., J. Vila, L. Gimferrer, M. Pinana, J. Esperalba, M. G. Codina, M. Barnes, M. C. Martin, F. Fuentes, S. Rubio, P. Alcubilla, C. Rodrigo, T. Pumarola, and A. Anton (2019), J. Clin. Virol. 110, 29.

Bal, A., M. Sabatier, T. Wirth, M. Coste-Burel, M. Lazrek, K. Stefic, K. Brengel-Pesce, F. Morfin, B. Lina, I. Schuffenecker, and L. Josset (2019), Euro Surveill. 24 (3).

Bedford, T., S. Riley, I. G. Barr, S. Broor, M. Chadha, N. J. Cox, R. S. Daniels, C. P. Gunasekaran, A. C. Hurt, A. Kelso, A. Klimov, N. S. Lewis, X. Li, J. W. McCauley, T. Odagiri, V. Potdar, A. Rambaut, Y. Shu, E. Skepner, D. J. Smith, M. A. Suchard, M. Tashiro, D. Wang, X. Xu, P. Lemey, and C. A. Russell (2015), Nature 523 (7559), 217.

Bottcher, S., C. Prifert, B. Weissbrich, O. Adams, S. Aldabbagh, A. M. Eis-Hubinger, and S. Diedrich (2016), Euro Surveill. 21 (19).

Centers for Disease Control and Prevention (CDC), (2011), MMWR. Morbidity and mortality weekly report 60 (38), 1301.

Cottrell, S., C. Moore, M. Perry, E. Hilvers, C. Williams, and A. G. Shankar (2018), Euro Surveill. 23 (46).

Du, J., B. Zheng, W. Zheng, P. Li, J. Kang, J. Hou, R. Markham, K. Zhao, and X.-F. Yu (2015), PloS one 10 (12), e0144208.

Dyrdak, R., M. Grabbe, B. Hammas, J. Ekwall, K. E. Hansson, J. Luthander, P. Naucler, H. Reinius, M. RotzénÖstlund, and J. Albert (2016), Eurosurveillance 21 (46).

Dyrdak, R., M. Mastafa, E. B. Hodcroft, R. A. Neher, and J. Albert (2019), Virus Evol 5 (1), vez007.

Funakoshi, Y., K. Ito, S. Morino, K. Kinoshita, Y. Morikawa, T. Kono, Y. H. Doan, H. Shimizu, N. Hanaoka, M. Konagaya, T. Fujimoto, A. Suzuki, T. Chiba, T. Akiba, Y. Tomaru, K. Watanabe, N. Shimizu, and Y. Horikoshi (2019), Pediatr Int 61 (8), 768.

Hadfield, J., C. Megill, S. M. Bell, J. Huddleston, B. Potter, C. Callender, P. Sagulenko, T. Bedford, and R. A. Neher (2018), Bioinformatics 10.1093/bioinformatics/bty407.

Harrison, C. J., W. C. Weldon, B. A. Pahud, M. A. Jackson, M. S. Oberste, and R. Selvarangan (2019), Emerging Infect. Dis. 25 (3), 585.

Hixon, A. M., J. Frost, M. J. Rudy, K. Messacar, P. Clarke, and K. L. Tyler (2019), Viruses 11 (9), 10.3390/v11090821
Holm-Hansen, C. C., S. E. Midgley, and T. K. Fischer (2016), The Lancet Infectious Diseases 16 (5), e64.

Imamura, T., M. Okamoto, S.-i. Nakakita, A. Suzuki, M. Saito, R. Tamaki, S. Lupisan, C. N. Roy, H. Hiramatsu, K.-e. Sugawara, K. Mizuta, Y. Matsuzaki, Y. Suzuki, and H. Oshitani (2014), Journal of Virology 88 (5), 2374.

Karelehto, E., G. Koen, K. Benschop, F. van der Klis, D. Pajkrt, and K. Wolthers (2019), Euro Surveill 24 (35), 10.2807/1560-7917.Es.2019.24.35.1800671

Katoh, K., K. Misawa, K.-i. Kuma, and T. Miyata (2002), Nucleic Acids Research 30 (14), 3059

Khetsuriani, N., A. Lamonte-Fowlkes, S. Oberst, M. A. Pallansch, and Centers for Disease Control and Prevention (2006), Morbidity and Mortality Weekly Report. Surveillance Summaries (Washington, D.C.: 2002) 55 (8), 1.

Kingman, J. F. C. (1982), Stochastic Processes and their Applications 13 (3), 235

Kramer, R., M. Sabatier, T. Wirth, M. Pichon, B. Lina, I. Schuffenecker, and L. Josset (2018), Euro Surveill. 23 (37).

Kroneman, A., H. Vennema, K. Deforche, H. Avoort, S. Penaranda, M. Oberste, J. Vinjé, and M. Koopmans (2011), Journal of Clinical Virology $\mathbf{5 1}$ (2), 121.

Kujawski, S. A., C. M. Midgley, B. Rha, J. Y. Lively, W. A. Nix, A. T. Curns, D. C. Payne, J. A. Englund, J. A. Boom, J. V. Williams, G. A. Weinberg, M. A. Staat, R. Selvarangan, N. B. Halasa, E. J. Klein, L. C. Sahni, M. G. Michaels, L. Shelley, M. McNeal, C. J. Harrison, L. S. Stewart, A. S. Lopez, J. A. Routh, M. Patel, M. S. Oberste, J. T. Watson, and S. I. Gerber (2019), MMWR Morb. Mortal. Wkly. Rep. 68 (12), 277.

Lau, S. K., C. C. Yip, P. S. Zhao, W. N. Chow, K. K. To, A. K. Wu, K. Y. Yuen, and P. C. Woo (2016), Sci Rep 6, 25147.

Levy, A., J. Roberts, J. Lang, S. Tempone, A. Kesson, A. Dofai, A. J. Daley, B. Thorley, and D. J. Speers (2015), Journal of Clinical Virology 69, 117.

Linsuwanon, P., J. Puenpa, K. Suwannakarn, V. Auksornkitti, P. Vichiwattana, S. Korkong, A. Theamboonlers, and Y. Poovorawan (2012), PloS one 7 (5), e35190.

Liu, Y., J. Sheng, A. Fokine, G. Meng, W.-H. Shin, F. Long, R. J. Kuhn, D. Kihara, and M. G. Rossmann (2015a), Science 347 (6217), 71.

Liu, Y., J. Sheng, A. Fokine, G. Meng, W.-H. Shin, F. Long, R. J. Kuhn, D. Kihara, and M. G. Rossmann (2015b), Science (New York, N.Y.) 347 (6217), 71

Majumdar, M., T. Wilton, Y. Hajarha, D. Klapsa, and J. Martin (2019), bioRxiv , 738948

Messacar, K., K. Pretty, S. Reno, and S. R. Dominguez (2019), J. Clin. Virol. 113, 24.

Mishra, N., T. F. F. Ng, R. L. Marine, K. Jain, J. Ng, R. Thakkar, A. Caciula, A. Price, J. A. Garcia, J. C. Burns, K. T. Thakur, K. L. Hetzler, J. A. Routh, J. L. KonopkaAnstadt, W. A. Nix, R. Tokarz, T. Briese, M. S. Oberste, and W. I. Lipkin (2019), mBio 10 (4), e01903

Nee, S., E. C. Holmes, A. Rambaut, and P. H. Harvey (1995), Philosophical Transactions of the Royal Society of London. Series B: Biological Sciences 349 (1327), 25

Nguyen, L.-T., H. A. Schmidt, A. von Haeseler, and B. Q. Minh (2015), Molecular Biology and Evolution 32 (1), 268.

Nix, W. A., M. S. Oberste, and M. A. Pallansch (2006), J. Clin. Microbiol. 44 (8), 2698.

Pellegrinelli, L., F. Giardina, G. Lunghi, S. C. Uceda Renteria, L. Greco, A. Fratini, C. Galli, A. Piralla, S. Binda, 
E. Pariani, and F. Baldanti (2019), Euro Surveill. 24 (7).

Petrova, V. N., and C. A. Russell (2018), Nature Reviews Microbiology 16 (1), 47.

Pickett, B. E., E. L. Sadat, Y. Zhang, J. M. Noronha, R. B. Squires, V. Hunt, M. Liu, S. Kumar, S. Zaremba, Z. Gu, et al. (2011), Nucleic acids research 40 (D1), D593.

Piralla, A., A. Girello, M. Grignani, M. Gozalo-Margüello, A. Marchi, G. Marseglia, and F. Baldanti (2014), Journal of medical virology $\mathbf{8 6}$ (9), 1590.

Poelman, R., E. H. Schölvinck, R. Borger, H. G. Niesters, and C. van Leer-Buter (2015a), Journal of Clinical Virology 62, 1 .

Poelman, R., I. Schuffenecker, C. Van Leer-Buter, L. Josset, H. G. Niesters, B. Lina, E.-E. E.-D. study group, et al. (2015b), Journal of Clinical Virology 71, 1.

Pons-Salort, M., M. S. Oberste, M. A. Pallansch, G. R. Abedi, S. Takahashi, B. T. Grenfell, and N. C. Grassly (2018), Proc. Natl. Acad. Sci. U.S.A. 115 (12), 3078.

$\mathrm{R}$ Core Team, (2014), $R:$ A Language and Environment for Statistical Computing, R Foundation for Statistical Computing, Vienna, Austria.

Rahamat-Langendoen, J., A. Riezebos-Brilman, R. Borger, R. van der Heide, A. Brandenburg, E. Schölvinck, and H. G. Niesters (2011), Journal of Clinical Virology 52 (2), 103.

Sagulenko, P., V. Puller, and R. A. Neher (2018), Virus Evolution 4 (1), 10.1093/ve/vex042.

Schieble, J. H., V. L. Fox, E. H. Lennette, et al. (1967), American journal of epidemiology 85 (2), 297.

Schrödinger, LLC, (2015), "The PyMOL molecular graphics system, version $2, "$.

Schuffenecker, I., A. Mirand, L. Josset, C. Henquell, D. Hecquet, L. Pilorgé, J. Petitjean-Lecherbonnier, C. Manoha, J. Legoff, C. Deback, et al. (2016), Eurosurveillance 21 (19).

Sedlazeck, F. J., P. Rescheneder, and A. von Haeseler (2013), Bioinformatics 29 (21), 2790.

Shen, L., C. Gong, Z. Xiang, T. Zhang, M. Li, A. Li, M. Luo, and F. Huang (2019), Scientific reports 9 (1), 6073.

Smura, T., P. Ylipaasto, P. Klemola, S. Kaijalainen, L. Kyllönen, V. Sordi, L. Piemonti, and M. Roivainen (2010), Journal of medical virology 82 (11), 1940.

Sun, S., F. Gao, Y. Hu, L. Bian, X. Wu, Y. Su, R. Du, Y. Fu, F. Zhu, Q. Mao, and Z. Liang (2018), Emerging Microbes \& Infections 7 (1), 99.

Tokarz, R., C. Firth, S. A. Madhi, S. R. Howie, W. Wu, S. Haq, T. Briese, W. I. Lipkin, et al. (2012), Journal of general virology 93 (9), 1952.

Uprety, P., D. Curtis, M. Elkan, J. Fink, R. Rajagopalan, C. Zhao, K. Bittinger, S. Mitchell, E. R. Ulloa, S. Hopkins, and E. H. Graf (2019), Emerg Infect Dis 25 (9), 1676

Vogt, M. R., and J. E. Crowe Jr (2018), Journal of the Pediatric Infectious Diseases Society 7 (suppl_2), S49.

Wisdom, A., E. C. Leitch, E. Gaunt, H. Harvala, and P. Simmonds (2009), J. Clin. Microbiol. 47 (12), 3958.

Wollants, E., L. Beller, K. Beuselinck, M. Bloemen, K. Lagrou, M. Reynders, and M. Van Ranst (2019), J. Clin. Virol. 121, 104205.

Xiang, Z., L. Li, L. Ren, L. Guo, Z. Xie, C. Liu, T. Li, M. Luo, G. Paranhos-Baccala, W. Xu, and J. Wang (2017), Emerg Microbes Infect 6 (5), e32 


\section{SUPPLEMENTARY MATERIALS AND METHODS}

\section{A. Patient population/samples.}

Respiratory samples positive for EV-D68 were collected from six virology laboratories (see Table II). Ethical approvals and consents were obtained as required by local regulations (see below).

In Stockholm, EV-D68 testing was done on all respiratory samples positive for enterovirus from 1 August to 31 October 2018 at the Karolinska University Laboratory, which serves 6 of 8 emergency hospitals in the Stockholm county. An additional 25 enterovirus-positive samples from the pediatric ICU at Karolinska University Hospital collected during November 2018 were also tested. Specific EV-D68 testing was done using a published realtime PCR (Dyrdak et al., 2016). All samples positive with an in-house EV-D68-specific qPCR (Dyrdak et al. 2016) were included. Eluates were obtained from extraction on an automated system (MagNA Pure [Roche]). RNA extraction of samples shipped to Stockholm from the other study centers was done by manually using the RNeasy Lipid Tissue Mini Kit (Qiagen cat. No. 74804). The study was reviewed and approved by the Regional Ethical Review Board in Stockholm, Sweden (registration no. 2017/1317-32).

In Groningen, testing was done routinely (year around) using a specific assay for rhinovirus or enterovirus, or a combination of rhinovirus/enterovirus (FilmArray RP2, BioFire). If positive, a specific enterovirus D68 assay was used. A selection of 10 samples with low Ct-values of a total of 21 positive samples in 2018 were sent to Stockholm (Ethical Approval METc 2017/278).

In Belgium, for the first time, there was an upsurge of EV-D68 in 2018 (Wollants et al. 2019). At Leuven, 10 positive EV-D68 samples with low Ct-values, sent from AZ Sint. Jan Brugge, were confirmed with a nested VP4/VP2 PCR (Wisdom et al., 2009). This hospital in Bruges detected 83 positive EV-D68 samples in 7,986 respiratory samples in 2018 by using TAC (Taqman Array Card) technology for broad respiratory screening. In 2015 a specific real-time PCR for EVD-68 was integrated on this microarray card (Poelman et al. $2015 \mathrm{a}$ ). In the context of a national reference laboratory for enteroviruses, no ethical approval was needed.

In Basel, 157 samples were screened using an in-house PCR based on primers by Piralla et al. (2014). The six samples sent to sent to Stockholm represent all positive samples. The catchment area of the laboratory did not include pediatric departments. For University Hospital Basel samples, only anonymized samples without additional patient data were used, which does not require a specific ethical evaluation in accordance with correspondence with Swissethics.

In Barcelona, detection of EV in respiratory specimens was performed by specific real-time multiplex RT-PCR assay (Allplex Respiratory Panel Assay, Seegene, Korea). EV were characterized by phylogenetic analyses based on VP1 sequence, as previously described (Andres et al. , 2019). A selection of 14 positive samples with low Ct-values of a total of 44 EV-D68 positive specimens from 2018 were used in this study. Institutional Review Board approval (PR(AG)173/2017) was obtained from the HUVH Clinical Research Ethics Committee.

In Tenerife, primary detection of enterovirus was done by specific real-time multiplex RT-PCR assay (Allplex Respiratory Panel Assay, Seegene, Korea) with subsequent typing of positive samples by VP1 sequencing (Nix et al., 2006). Consent for further analysis was obtained for nine of a total of twelve positive samples. Eluates of the nine samples, obtained from extraction on an automated system (EasyMag-EMag [Biomerieux]), were sent to Stockholm. Local Research Ethics Committee approval was obtained (code CHUNSC_2019_02).

\section{B. Sequencing and bioinformatic processing}

Near full-length genome sequencing ('whole genome' sequencing) was performed as previously described (Dyrdak et al. 2019). Briefly, the genome was amplified in duplicate by one-step RT-PCR in four overlapping fragments. Duplicates of each fragment were pooled and purified using AGENCOURT AMPure XP PCR purification kit and quantified with Qubit assays (Q32851, Life Technologies). Purified DNA from each fragment was diluted to the same concentration, pooled and sent to the Clinical Genomics Unit at Science for Life Laboratory for library preparation and sequencing (SciLifeLab, Stockholm, Sweden).

In total $1 \mu \mathrm{l}$ of DNA $(\sim 0.5-2.0 \mathrm{ng} / \mu \mathrm{l})$ was used in the tagmentation reaction using Nextera chemistry (Illumina) to yield fragments $>150 \mathrm{bp}$. The tagmented library underwent eleven cycles of PCR with single-end indexed primers (IDT Technologies) followed by purification using Seramag beads. The library was quantified using Quant-iT dsDNA High-Sensitivity Assay Kit and Tecan Spark 10 M or FLUOstar Omega plate reader. The library was then pair-end sequenced to a depth of $100,000-1,500,000$ reads per sample on either HiSeq $2500(2 \times 101 \mathrm{bp})$ or NovaSeq $6000(2 \times 151 \mathrm{bp})$ Illumina sequencers. Base calling and demultiplexing was done using bcl2fastq v1.87, without allowing any mismatch in the index sequence. Assembly was done as described previously (Dyrdak et al., 2019), but to improve mapping sensitivity, we replaced BWA by NextGenMap (Sedlazeck et al. , 2013) and used mapping references from the same subclade as the sample. The scripts implementing this workflow are available on github at github.com/neherlab/EV-D68_sequence_mapping. Fiftytwo of 55 samples were sequenced with a coverage of $>100 \times$ in all four fragments and were included in the 
further analysis along with one sample with a coverage of $>10 \times$ in one fragment and $>100 \times$ in the other three fragments, giving a total of 53 successfully sequenced samples. The consensus sequences for these 53 samples have been deposited in GenBank (accession numbers MN245396-MN245448). The raw reads have been deposited in the Short Read Archive (BioProject number PRJNA525063, BioSample accession numbers SAMN13745166-SAMN13745216). A list of accession numbers, along with metadata is available as Supp. Table I.

\section{Whole Genome and VP1 Sequence Data Sets}

The consensus sequences from the 53 samples sequenced in this study were combined with whole genomes with length >6000bp available in the Virus Pathogen Resource (ViPR) (Pickett et al., 2011) (as of 2019-09-12) as well as samples matching this criterion manually curated from GenBank. Of all the sequences available in GenBank ( $n=4,259$, on 1 Nov 2019), 70\% were annotated with an isolation source, of which the majority were respiratory specimens $(88 \%)$.

To conduct additional analyses on an as large and representative a data set as possible, a further data set of VP1 sequences was assembled. All EV-D68 sequences in ViPR were downloaded and BLASTed (Altschul et al. , 1990) against a 927 bp reference VP1 alignment (KX675261). Only matching regions of at least $700 \mathrm{bp}$ and an Expect Value (E-value) of 0.005 or lower were included. All sequences from the whole genome data set were included in the VP1 dataset.

To counter over-representation of countries with high sample numbers during some time periods, the VP1 dataset was down-sampled using the 'augur' 'filter' command, randomly selecting at most 20 samples per month, per year, per country.

Though the 3 whole genome and 7 VP1 sequences sampled prior to 1990 fit the estimated molecular clock well when included in the analyses, they were omitted for figure clarity. Samples without a year of sampling were also excluded ( 3 from the whole genome run; 6 from the VP1 run), as well as mouse-adapted samples and extreme outliers (6 from the whole genome run; 31 from the VP1 run). Using 'augur' 'refine' to generate time-resolved trees, branches more then 5 interquartile distances from the substitution rate regression were pruned, removing 3 and 4 sequences from the whole genome and VP1 datasets. The final number of sequences included the whole genome and VP1 phylogenies was 813 and 1,654, respectively. Accession numbers and author/publication details for each sequence included in the analyses are available at nextstrain.org/enterovirus/d68/genome and nextstrain.org/enterovirus/d68/vp1. A list of accession numbers, along with metadata is available as Supp. Ta- bles III and IV.

\section{Age Data}

In order to analyze associations between and patient age and EV-D68 clade and subclade, roughly 500 ages or age-ranges were manually scraped from over 40 papers. Over 100 additional ages were provided by authors to whom we reached out. Combined with age data available on GenBank, this resulted in approximately 900 VP1 sequences and over 450 whole genome sequences with some kind of age information.

As some age information was available only as an agerange, age data was automatically parsed to create an age range variable. 'Age' contains the exact decimal year, where available, and 'age range 1' consists of four categories (<1yr, 1-5yrs, 6-17yrs, 18-64yrs, and >=65yrs). For the borderline cases of age ranges given as ' $0-1$ ' and ' $0-18$ ', these were interpreted as $<1$ yr and $<18$ yrs, respectively. Data was available for exact age and 'age range 1' for 778 and 792 VP1 samples and 378 and 378 whole genome samples.

The effect of subclade designation on age was examined using the $\mathrm{lm}$ function in $\mathrm{R}$ ( $\mathrm{R}$ Core Team, 2014) to perform simple linear models.

\section{E. Phylogenetic analysis}

We used the augur pipeline (Hadfield et al., 2018$)$ to analyze the whole genome and VP1 data sets. Briefly, sequences were aligned using mafft (Katoh et al., 2002) and annotated according to the 1962 Fermon strain (GenBank accession AY426531), a phylogenetic tree was inferred using IQ-TREE (Nguyen et al., 2015), and maximum likelihood time trees were inferred using TreeTime (Sagulenko et al., 2018). Samples deviating from the estimated clock rate by more than 5 inter-quartile distances were removed during this step. Classification into clades and subclades was automated using the augur 'clades' command, based on mutations which matched the typing assigned to sequences by the Enterovirus Genotyping Tool 0.1 at https: //www.rivm.nl/mpf/typingtool/enterovirus/ (Kroneman et al. 2011) (see Supp. Tables V and $\mid$ VI for mutations used to classify). The scripts implementing this workflow are available on github at github.com/nextstrain/enterovirus_d68. The scripts to produce the further analyses and figures for this paper are available at github.com/neherlab/2018_evd68_paneurope_analysis. 


\section{F. Diversification, Persistence, and Migration}

To calculate the number of lineages leading to the samples taken during each season, a season-specific tree was created from the time-resolved, whole genome phylogeny, which only contained tips sampled during the outbreak year under investigation. Trees were assumed to be ultrametric, so the number of lineages at the end of the season is equal to the number of samples taken that year. Working backwards from the most recent sample, each coalescence event and the time is occurred was recorded, until only one lineage remained. To help show the similarity in lineage change between the seasons, they are plotted as years prior to the end of the ourbreak year in Fig. $2 \mathrm{~B}$.

Maximum likelihood estimates of migration rates were performed with TreeTime v0.7.0 using the command-line interface as explicitly documented in the Snakefile. In order to minimize bias by countries, regions, and years with very few samples, we estimated migration rates from VP1 trees pruned to only include tips from the outbreak year (2014, 2016, or 2018) under investigation, and metadata was masked to include only countries (in Europe) and regions with a relatively similar number of samples across outbreaks. For between-country migration estimates the countries were thus France, Spain, Germany, Italy, Sweden, "rest of Europe", and "rest of world", and for regions China, Europe, North America, and "rest of world." However, estimates did not differ greatly from when all tips, countries, and regions were used. Similarly, estimates of the coalescent rate through time (aka "skyline") was performed with TreeTime using $n=150$ bins and restricting to full genome sequences after Jan 2011.

To color the VP1 tree by epitope patterns, amino acids at the specified locations were concatenated, and only those appearing more than 7 times (6 times for the $\mathrm{C}$ terminus) were displayed. The C-terminus was not included in a large fraction of sequences resulting in many undetermined amino acids. Tips with more than 7 missing amino acid positions were grouped into a 'many $\mathrm{X}$ ' category; missing amino acids were inferred from the parental sequence for patterns with 6 or fewer missing sites. The crystal structure in Fig. 4 was generated using PyMOL (Schrödinger, LLC, 2015). 


\section{Sample Date of 53 Included 2018 Samples}

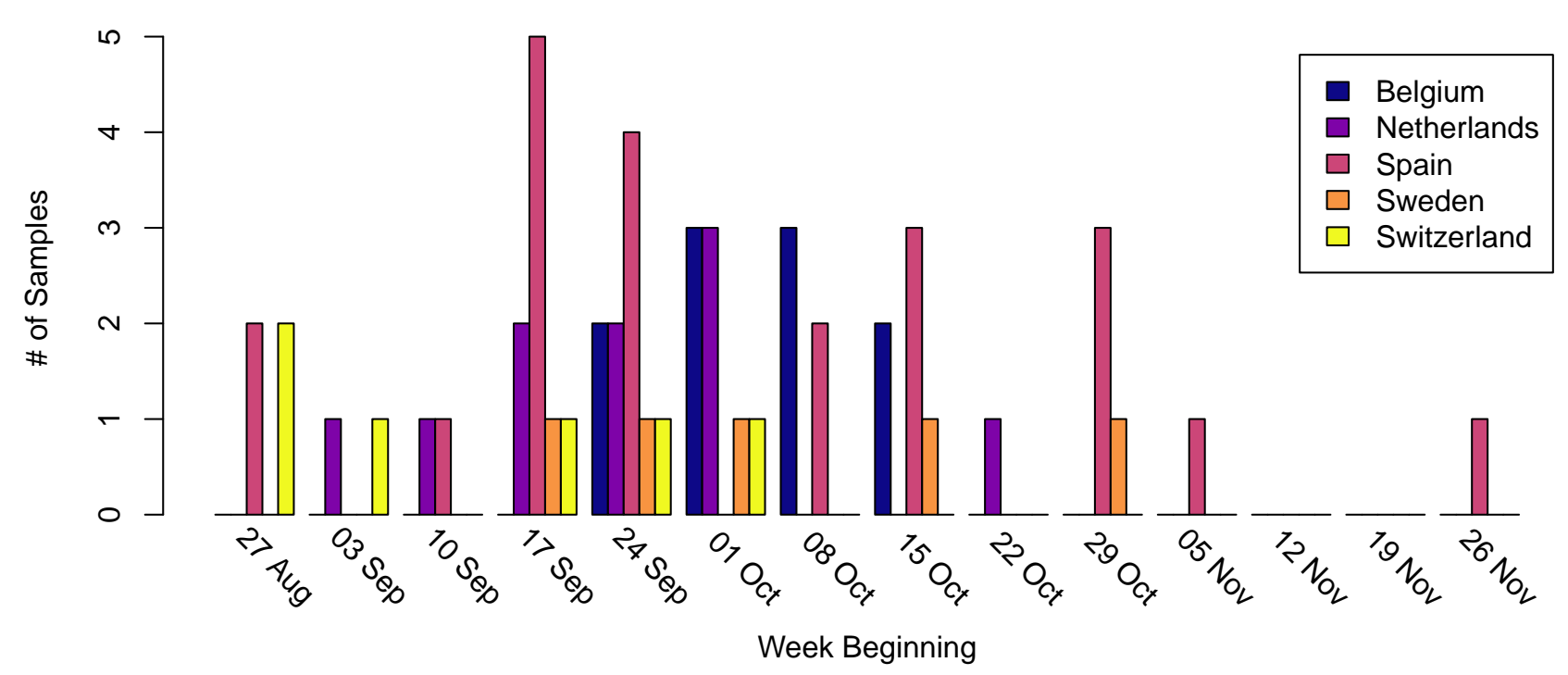

Figure S 1 Histogram of the sampling dates, by country, for the 53 samples generated in this study that were included in the final analyses.

Table S II AA Mutations in BC and DE Loops, C-Terminus, and VP2. This table extends from Fig 4 panel B, but includes the pattern differences for the C-terminus and VP2 AA positions 135-156.

\begin{tabular}{|c|c|c|c|c|}
\hline Strain & BC loop & DE loop & C-Terminus & VP2 \\
\hline Fermon & . A..S.G. & . . NND. & K.R.T.........A.T.....X & HD.T...G......R...N \\
\hline root & NHTSSDARTHKNFF & NGSSNSTYM & RGKDRAPNTLNAIIGNRESVKTMPHNIVTT & YNTNTSPEFNDIMKGEEGGTFN \\
\hline A & $\ldots$....D... & $-\ldots$ & 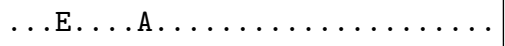 & $\ldots \ldots \ldots \mathrm{T} \ldots \ldots \ldots \mathrm{S}$ \\
\hline $\mathrm{A} 2$ & $\ldots$....VD...Y & $-\ldots N \ldots$ & K..E...A........ I...D. .N. & H. .T...G.D... \\
\hline A1 & ..A.E.Q.D... & $-\mathrm{S} \ldots \mathrm{T}$ & ..E.......... D. & .D.T..G.T.......S \\
\hline B & D...A.Q.D... & $\ldots$..... & K..E...A..... D. & ....G.D. . \\
\hline B3-US & D...T.Q.D... & $\ldots$. .N..V & K. E...A...... D. & H..T...G.D.....A. \\
\hline B3-EU & D....A.QAD. . & $\ldots$....V & K. E. A A. W DD. & H..T...G.D....A. \\
\hline
\end{tabular}




\section{Age Distribution by Clade of 100 Bootstraps}

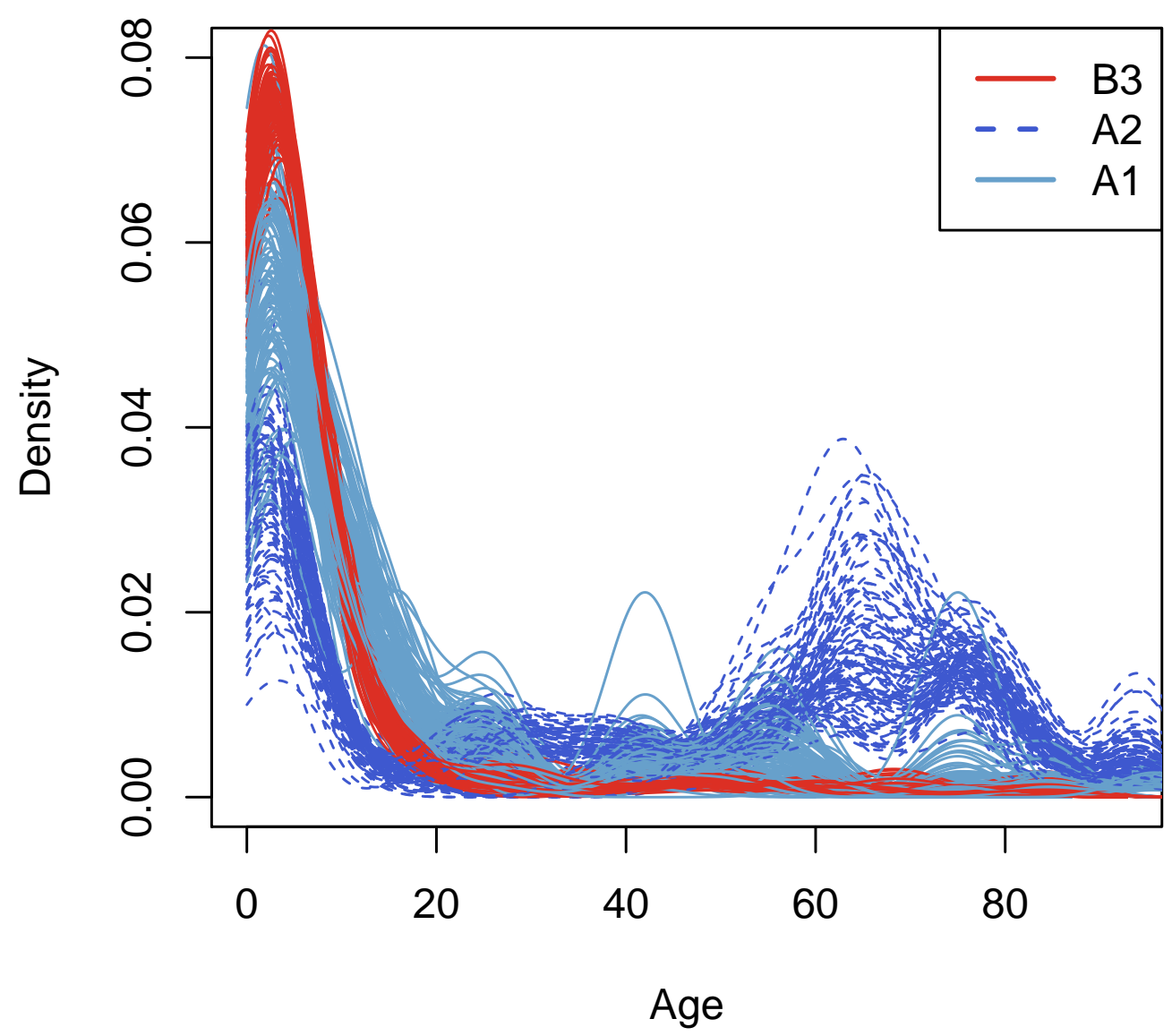

Figure S 2 For the VP1 dataset, samples were sampled with replacement by country 100 times. For each bootstrapped dataset, the same linear regression shown in Table I was performed, and the age distributions for the A1, A2, and B3 clades was plotted. The ages of the A2 clade was significantly different from the B3 clade in all bootstrap replicate linear regressions after Bonferroni correction. 
bioRxiv preprint doi: https://doi.org/10.1101/2020.01.10.901553; this version posted January 18, 2020. The copyright holder for this preprint (which was not certified by peer review) is the author/funder. All rights reserved. No reuse allowed without permission.

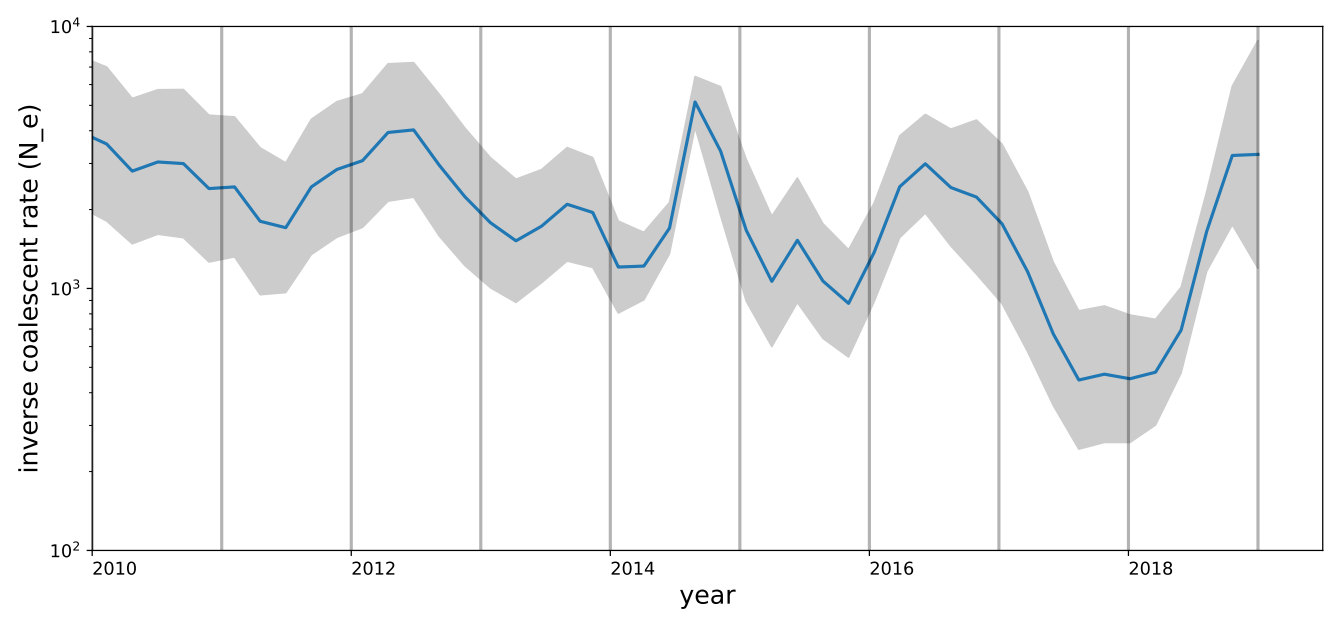

Figure S 3 Inferred inverse rate of coalescence per lineage through time.

Table S V Clade definitions for VP1 dataset

\begin{tabular}{|c|c|c|}
\hline Clade & Nucleotide Position & Base \\
\hline \multirow{3}{*}{ A } & 207 & A \\
& 291 & A \\
& 834 & $\mathrm{C}$ \\
\hline \multirow{3}{*}{ A1 } & 201 & $\mathrm{~T}$ \\
& 533 & $\mathrm{C}$ \\
& 891 & $\mathrm{~T}$ \\
\hline & 132 & $\mathrm{~T}$ \\
& 201 & $\mathrm{~T}$ \\
& 345 & $\mathrm{G}$ \\
\hline & 198 & $\mathrm{~A}$ \\
$\mathrm{~B}$ & 390 & $\mathrm{G}$ \\
& 807 & $\mathrm{G}$ \\
\hline \multirow{2}{*}{ B1 } & 687 & $\mathrm{~T}$ \\
& 702 & $\mathrm{~T}$ \\
\hline \multirow{3}{*}{ B2 } & 132 & $\mathrm{~A}$ \\
& 343 & $\mathrm{~T}$ \\
& 459 & $\mathrm{~A}$ \\
\hline & 102 & $\mathrm{~A}$ \\
B3 & 270 & $\mathrm{C}$ \\
& 468 & $\mathrm{G}$ \\
\hline \multirow{2}{*}{$\mathrm{C}$} & 684 & $\mathrm{~T}$ \\
& 798 & $\mathrm{C}$ \\
\hline
\end{tabular}


bioRxiv preprint doi: https://doi.org/10.1101/2020.01.10.901553; this version posted January 18, 2020. The copyright holder for this preprint (which was not certified by peer review) is the author/funder. All rights reserved. No reuse allowed without permission.

Table S VI Clade definitions for whole genome dataset

\begin{tabular}{|c|c|c|c|}
\hline Clade & Gene or Nucleotide & Position & Base \\
\hline \multirow{3}{*}{ A } & nuc & 156 & $\mathrm{~T}$ \\
& nuc & 333 & $\mathrm{~A}$ \\
& nuc & 675 & $\mathrm{G}$ \\
\hline \multirow{2}{*}{ A2 } & nuc & 204 & $\mathrm{~T}$ \\
& nuc & 3864 & $\mathrm{C}$ \\
\hline \multirow{3}{*}{ B } & nuc & 711 & $\mathrm{G}$ \\
& nuc & 721 & $\mathrm{C}$ \\
& nuc & 744 & $\mathrm{G}$ \\
& nuc & 942 & $\mathrm{~T}$ \\
B1 & nuc & 1062 & $\mathrm{C}$ \\
& nuc & 1122 & $\mathrm{~T}$ \\
\hline \multirow{3}{*}{ B2 } & nuc & 957 & $\mathrm{~A}$ \\
& nuc & 3090 & $\mathrm{~T}$ \\
& nuc & 4290 & $\mathrm{C}$ \\
\hline \multirow{2}{*}{ B3 } & nuc & 711 & $\mathrm{G}$ \\
& nuc & 999 & $\mathrm{C}$ \\
& nuc & 1038 & $\mathrm{C}$ \\
& nuc & 1137 & $\mathrm{~A}$ \\
\hline & nuc & 831 & $\mathrm{~T}$ \\
& nuc & 924 & $\mathrm{~A}$ \\
\hline & nuc & 26 & $\mathrm{R}$ \\
& nuc & 1299 & $\mathrm{~T}$ \\
& & & \\
\hline
\end{tabular}




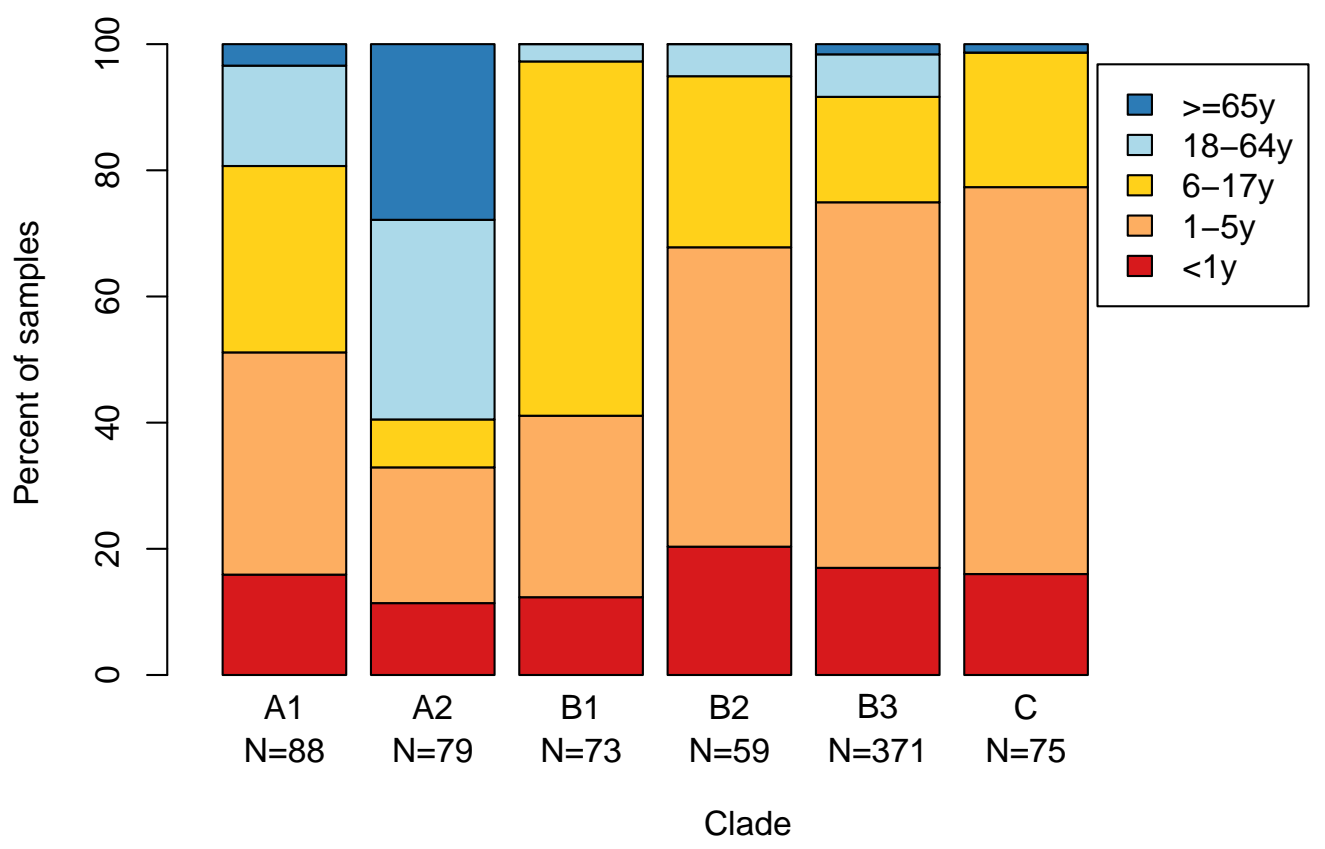

Figure S 4 For VP1 dataset samples with 'age_range1' data, for all years, the proportion of samples in each age category for each clade. The over-representation of adults and the elderly in the A2 subclade can be seen clearly, along with the over-representation of adults (and to a lesser extent, the elderly) in the A1 subclade. 
bioRxiv preprint doi: https://doi.org/10.1101/2020.01.10.901553; this version posted January 18, 2020. The copyright holder for this preprint (which was not certified by peer review) is the author/funder. All rights reserved. No reuse allowed without permission.
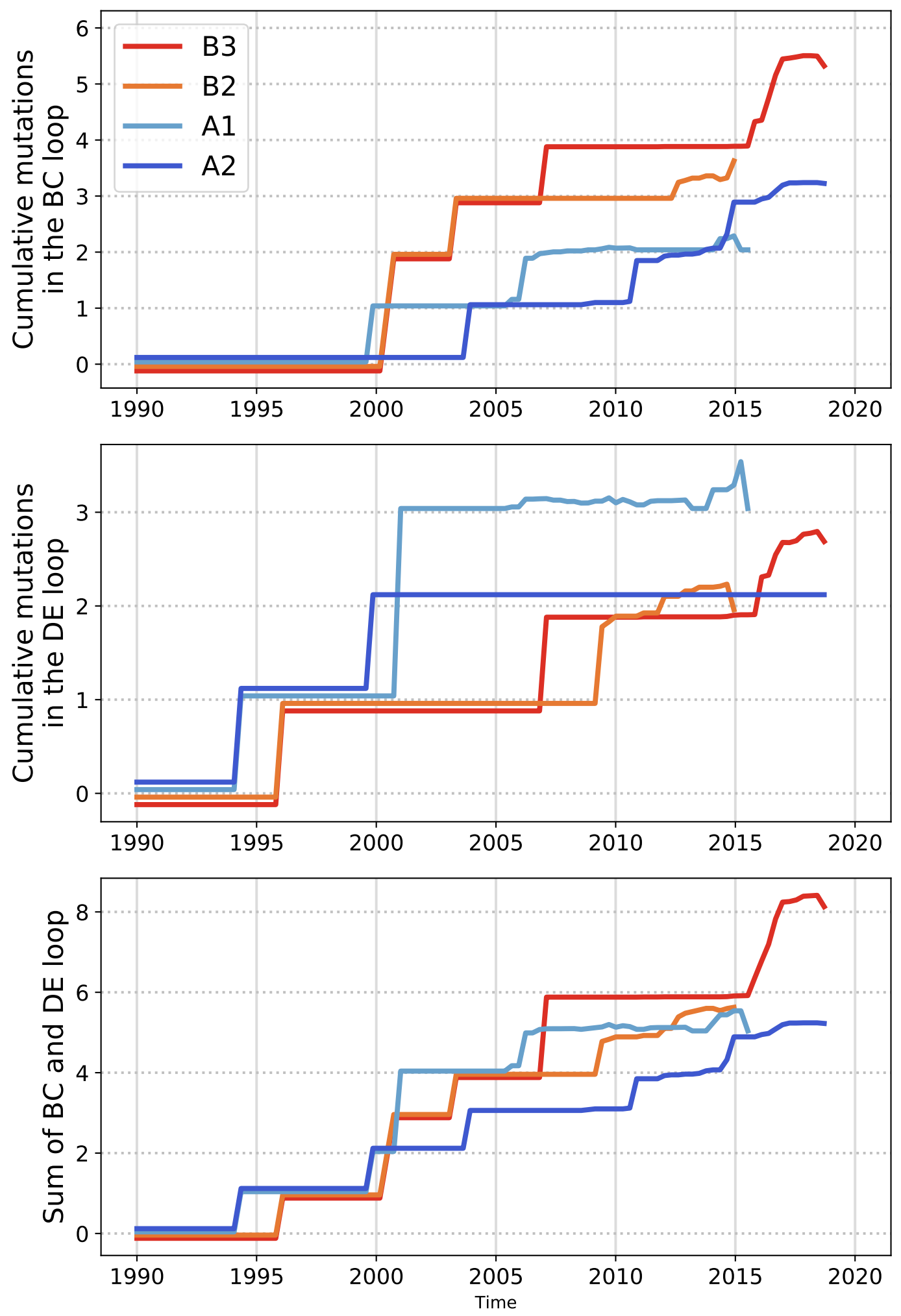

Figure S 5 Mean number of AA mutations in the BC and DE loops over time, colored by clade. In the BC loop, AA positions 90, 92, 95, 97, 98, and 103, were used, and in the DE loop, AA positions 140-146 and 149 were used. The BC-loop plot (top) shows that the B3 clade had around 4 mutations between 2000 and 2008, then about 7 years without mutations. In contrast, the A2 clade had only one mutation prior to 2010, but then had two between 2011 and 2015. Mean mutation count lines have been plotted slightly above and below their true value so that all lines can be seen when they share the same value. Calculated for the whole genome dataset. 
bioRxiv preprint doi: https://doi.org/10.1101/2020.01.10.901553; this version posted January 18, 2020. The copyright holder for this preprint (which was not certified by peer review) is the author/funder. All rights reserved. No reuse allowed without permission.

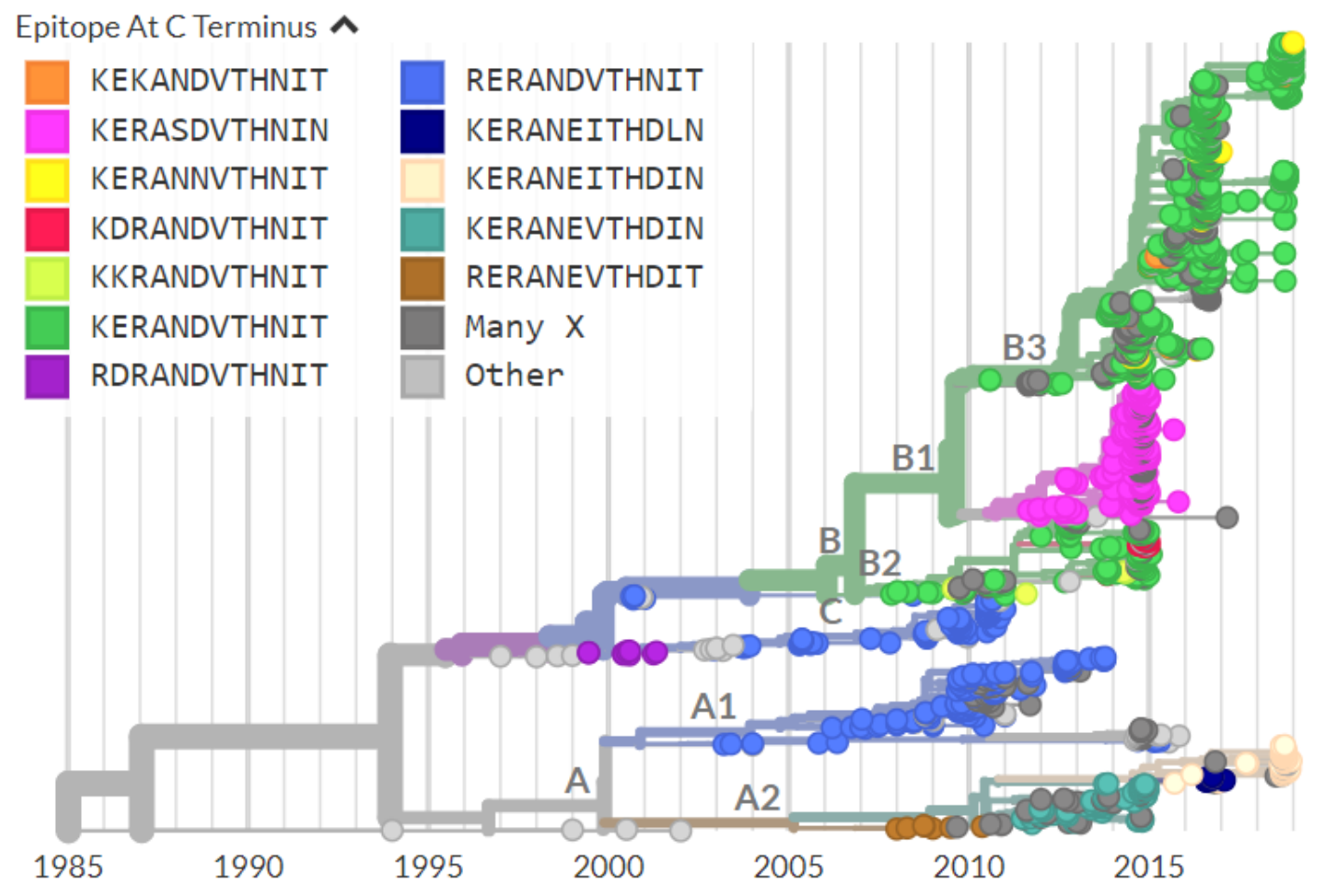

Figure S 6 Most common C-terminus epitope patterns on the VP1 tree. This figure extends from Fig 4 panels D \& E, but shows the most common C-terminus patterns. As in the BC-loop in Fig 4 the A2 subclade shows substantial recent evolution in this region. 